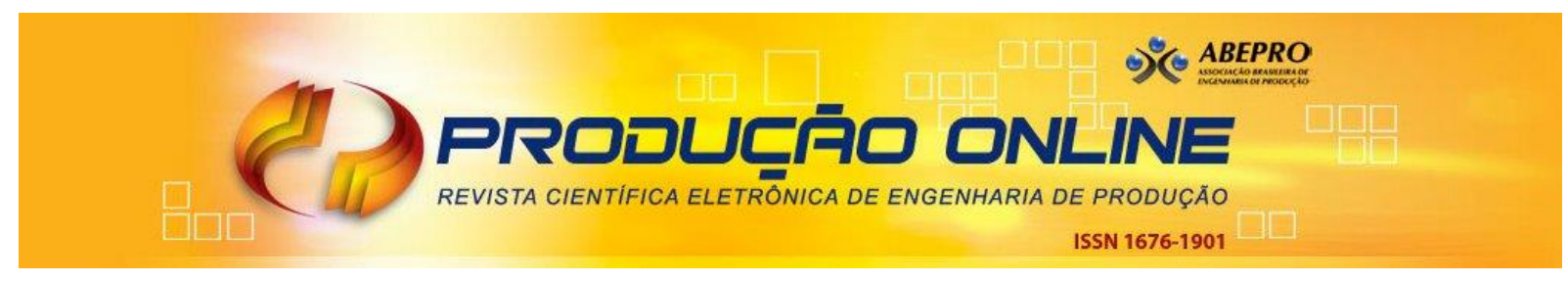

\title{
MÉTODOS QUANTITATIVOS PARA SELEÇÃO DE FORNECEDORES SUSTENTÁVEIS: UMA REVISÃO SISTEMÁTICA DA LITERATURA
}

\section{QUANTITATIVE METHODS FOR SUSTAINABLE SUPPLIER SELECTION: A SYSTEMATIC LITERATURE REVIEW}

\author{
Gilberto Miller Devós Ganga*E-mail: ganga@dep.ufscar.br \\ Letícia Reis Rodrigues* E-mail: Ireisrodrigues@hotmail.com \\ Rui Tadashi Yoshino**E-mail: ruiyoshino@utfpr.edu.br \\ Luis Antonio de Santa-Eulalia** E-mail: L.Santa-Eulalia@USherbrooke.ca \\ *Universidade Federal de São Carlos (UFSCar), São Carlos, SP, Brasil \\ **Universidade Tecnológica Federal do Paraná (UTFPR), Ponta Grossa, PR, Brasil \\ "Université de Sherbrooke, Canadá
}

\begin{abstract}
Resumo: A temática ambiental e social, cada vez mais presente e atuante no cotidiano das operações das empresas, precisa ser tratada no contexto da seleção de fornecedores, pois a literatura acadêmica e profissional é carente nesta área, especialmente no que tange ao uso de métodos quantitativos e no tratamento de aspectos sociais. Para tanto, o objetivo deste artigo foi realizar uma revisão sistemática da literatura com um propósito descritivo-explicativo que permitisse coletar, identificar, selecionar e avaliar criticamente os métodos quantitativos utilizados na seleção de fornecedores sustentáveis do ponto de vista verde e social. Buscando ir além de uma revisão sistemática tradicional, este trabalho fez uso também de técnicas de análise de conteúdo via tag clouds e de análise de correspondência. As principais evidências encontradas na literatura sobre seleção de fornecedores verdes/sustentáveis foi o uso intensivo de técnicas combinadas e a ampla adoção da lógica fuzzy nas técnicas multicritério, programação matemática e inteligência artificial.
\end{abstract}

Palavras-chave: Seleção de fornecedores verdes ou sustentáveis. Revisão sistemática da literatura. Análise de conteúdo. Análise de Correspondência.

Abstract: Both environmental and social issues are nowadays part of the decision-making process, but these aspects are not properly explored by the academic and professional literature about supplier selection. This is particularly true when considering the social perspective combined with quantitativeoriented selection methods. Thus, this paper presents a descriptive-explicative systematic literature review that collects, identifies, selects and critically analyzes several different quantitative methods for supplier selection, from both social and environmental viewpoints. In order to go beyond traditional literature reviews, this paper also employs both content analysis (via tag clouds) and correspondence method. Results indicate that the literature makes extensive use of combined approaches, mainly those merging fuzzy logic, multi-criteria, mathematical programming and artificial intelligence-based methods.

Keywords: Green or Sustainable Supplier selection. Systematic literature review. Tag cloud. Correspondence analysis. 


\section{INTRODUÇÃO}

A seleção de fornecedores é uma atividade de extrema importância na gestão do relacionamento com fornecedores, um processo de negócio chave na gestão da cadeia de suprimentos.

De modo idiossincrático, o advento da Gestão da Cadeia de Suprimentos Sustentável (CARTER; EASTON, 2011; SELLITO et al, 2013; SEURING; MILLER, 2008; SRIVASTAVA, 2007;) reforça a seleção de fornecedores sustentáveis como uma atividade que impacte diretamente no desempenho de empresas e de suas cadeias de suprimentos (TRACEY; TAN, 2001; ITTNER et al., 1999; KANNAN; TAN, 2006; NAIR; JAYARAM; DAS, 2015; VISWANADHAM; SAMVEDI, 2013).

A incorporação da dimensão ambiental (verde) e social, conjuntamente às perspectivas clássicas de desempenho econômico na seleção de fornecedores, tornam o processo de decisão mais complexo e multifacetado. Além da multiplicidade de critérios, ressalta-se a natureza diversa dos mesmos: ora qualitativo, ora quantitativo, além de outras características, tornando o processo de decisão um importante desafio aos gestores.

Nesse contexto, inúmeras abordagens vem sendo aplicadas na seleção de fornecedores sustentáveis. Destacam-se os métodos de decisão multicritério, programação matemática, inteligência artificial, além das abordagens híbridas combinando diferentes métodos.

Compreender o emprego destas técnicas de seleção de fornecedores sustentáveis à luz da literatura torna-se fundamental, dado que os trabalhos que buscaram organizar e sistematizar o conteúdo acadêmico e profissional na área ainda possuem limitações. Cabe ressaltar as seguintes: além de serem poucos os trabalhos, os mesmos possuem cobertura parcial e não utilizam de abordagens de análise de literatura mais sofisticadas, como os métodos descritivos de padrões relacionais, incluindo análise de conteúdo e de correspondência.

Para tanto, visando contribuir para reduzir tal lacuna de pesquisa, o objetivo deste artigo foi realizar uma revisão sistemática da literatura com um propósito descritivo-explicativo (GANGA, 2012) que permita coletar, identificar, selecionar e avaliar criticamente os métodos (ferramentas ou técnicas) que apoiem a seleção de fornecedores sustentáveis. Ademais, este trabalho vai além de outros estudos que contribuíram para uma sistematização parcial da literatura (GOVINDAN et al., 2015; ZHANG; ABDUL HAMID; THOO, 2014; IGARASHI; DE BOER; FET, 2013; CHAI; LIU; NGAI, 2013; HO; XU; DEY, 2010), pois utilizou-se da análise de conteúdo via tag cloud (SINCLAIR; CARDEW-HALL, 2008) e da análise de correspondência (SALAMIN; HANAPPI, 2014). Espera-se, assim, que este trabalho contribua na identificação de lacunas e na proposição de direcionadores de pesquisas futuras na seleção de fornecedores verdes/sustentáveis.

O artigo foi estruturado da seguinte forma. A Seção 2 discute conceitos termos-chave imprescindíveis à compreensão deste trabalho. A Seção 3 descreve 
os procedimentos adotados na realização da revisão sistemática de literatura. A Seção 4 apresenta os resultados encontrados, e finalmente a Seção 6 apresenta as considerações finais da pesquisa.

\section{FUNDAMENTAL TEÓRICO}

\subsection{Cadeias de Suprimentos Verde e Sustentável}

Os primeiros ${ }^{1}$ trabalhos publicados em periódicos relacionando cadeia de suprimentos e a abordagem ambiental, denotada neste trabalho por "verde" datam do período de 1994 a 1999 (WEBB, 1994; VAN HOEK, 1999; RIEBEL, 1999; SMITH, 1998). O trabalho de Weeb (1994) evidenciou, por exemplo, a consideração de critérios ambientais como parâmetros de compra de matéria prima. O termo Gestão da Cadeia de Suprimentos verde (green supply chain management) aparece pela primeira como artigo de periódico em 2003, com o trabalho de Sarkis (2003). Neste e nos demais trabalhos que adotaram a temática da questão ambiental (verde) no âmbito da gestão da cadeia de suprimentos, o foco era a busca de vantagens competitivas por meio da melhoria do desempenho ambiental sem comprometer os resultados econômicos ou operacionais da empresa ou da cadeia imediata em que estava inserida. O primeiro trabalho que aparece o termo "sustainable supply chain" é o de Stevenson, Jones e Macrae (2002) que discute problemas no processo de abastecimento considerando uma perspectiva em termos de desempenho mais ampla. Nesse sentido, o termo "sustentável", mais abrangente, incorpora além da perspectiva ambiental ou verde, as perspectivas econômica e social (NORMAN; MACDONALD, 2004; HACKING; GUTHRIE, 2008; HUBBARD, 2009), fazendo alusão ao conceito do triple botton line (ELKINGTON, 1998). A literatura levantada nas bases de dados considerou tanto a abordagem singular "verde", denotando preocupação à questão ambiental, como uma perspectiva mais ampla, considerando o termo sustentabilidade na seleção de fornecedores. Contudo, como será evidenciado nos resultados alcançados, existe uma predominância de trabalhos que focam predominantemente a temática ambiental (verde), também ressaltado por Seuring e Müller (2008). Neste trabalho será utilizada sempre a expressão "sustentável", procurando enfatizar as duas ênfases nos artigos pesquisados nas bases de dados.

\subsection{Compras e Seleção de Fornecedores Verde/Sustentável}

Hervani e Sarkis (2005) definem a gestão da cadeia de suprimentos verde como a incorporação da temática ambiental (verde) nas práticas, processos ou

1 Buscas nas base de dados SCOPUS, Web of Science e Engineering Village, usando como strings de busca o termo "green supply chain", somente em artigos de periódicos e nos títulos destes. 
atividades relativos à compras, manufatura, administração de materiais, marketing \& distribuição e a logística reversa. Neste trabalho adotamos o mesmo conceito de Hervani e Sarkis (2005), porém considerando além da perspectiva ambiental, a visão mais abrangente denotada pelo termo "sustentável". Além disso, consideramos, a forte relação entre compras verde e a seleção de fornecedores, ora como efeito de compras sobre a seleção de fornecedor (MIN; GALLE, 2001), ora a seleção de fornecedor como uma das atividades de compras verde (ZSIDISIN; SIFERD, 2001; LIN; CHEN; TING, 2012). A Figura 1 ilustra o modelo teórico-conceitual que relaciona gestão da cadeia de suprimentos verde/sustentável, compras verde/sustentável e seleção de fornecedores verde/sustentável.

Figura 1 - Quadro conceitual adotado neste trabalho

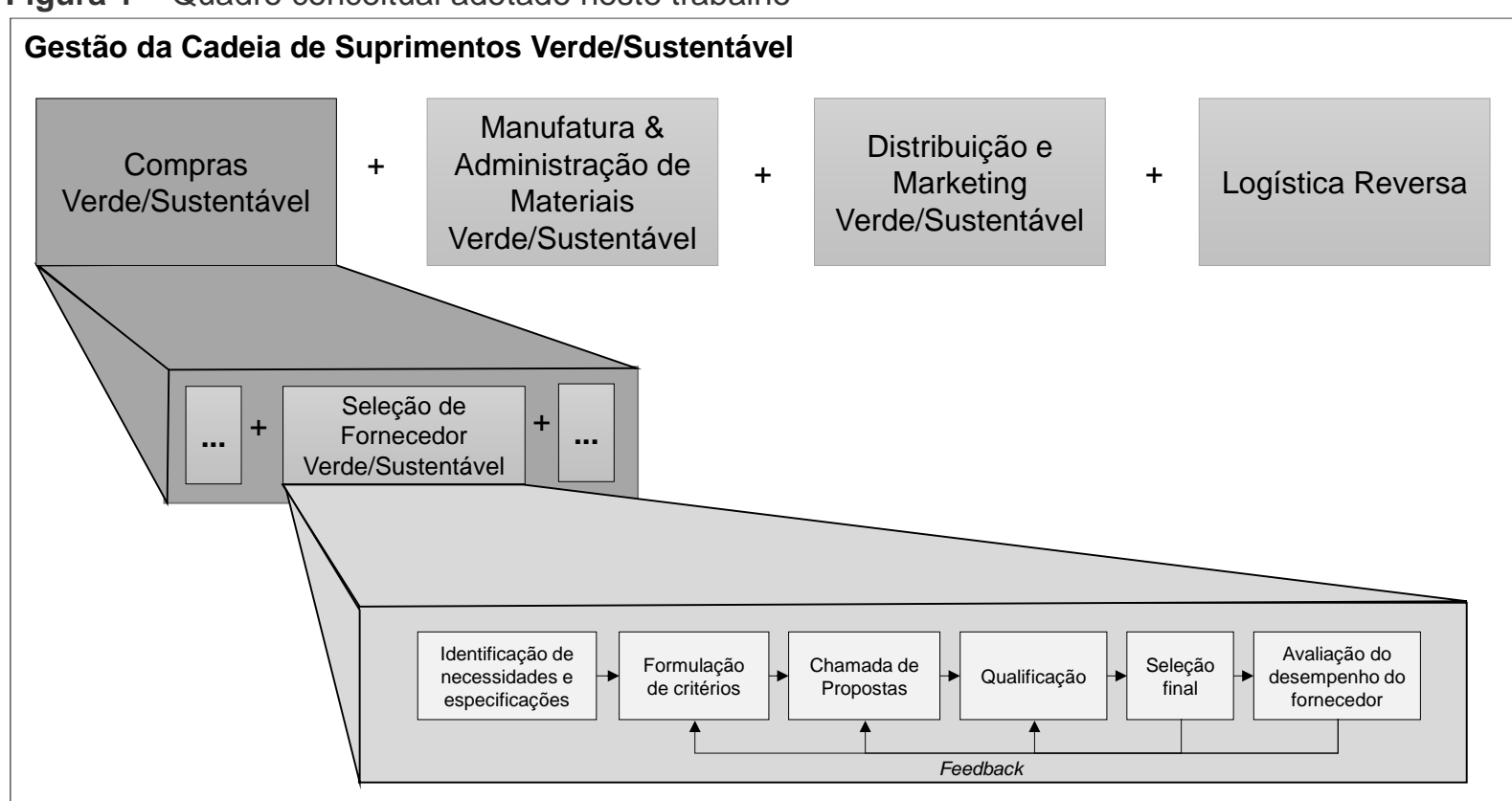

Fonte: Proposto pelos autores a partir de Hervani, Helms e Sarkis (2005), Min e Galle (2001), Zsidisin e Siferd (2001), Lin, Chen e Ting (2012) e Igarashi, De Boer e Fet (2013).

A seleção de fornecedores é composta de um conjunto de atividades, que varia de empresa para empresa, mas geralmente ela se inicia pelo processo de identificação de necessidades. Após os compradores definirem os critérios de desempenho dos fornecedores potenciais, uma chamada é realizada junto aos mesmos. A seleção é realizada após o envio e a revisão da documentação enviada pelos candidatos à fornecedores. Nesta etapa, geralmente ocorrem várias iterações, e a escolha final é feita a partir de um número de fornecedores pré-qualificados. Em algumas empresas, pode ocorrer uma avaliação posterior do desempenho dos fornecedores (COUSINS et al., 2008; De BOER; LABRO; MORLACHI, 2001; VAN WEELE, 2010 apud IGARASHI; De BOER; FET, 2013). 


\subsection{Métodos quantitativos que suportam a seleção de fornecedores}

Inúmeros métodos vem sendo utilizados para a seleção de fornecedores (De BOER; LABRO; MORLACHI, 2001). De Boer, Wegen e Telgen (1998) destaca que na fase de qualificação e seleção final são utilizadas ferramentas quantitativas, o foco deste trabalho. O autor destaca como classes de métodos, os modelos de ponderação linear (o AHP, por exemplo), modelos baseados no custo total de propriedade, modelos baseados em programação matemática, modelos estatísticos, modelos baseados em inteligência artificial.

Ho, Xu e Dey (2010) por sua vez classificam os métodos em abordagens individuais e integradas. Nas abordagens individuais os autores destacam os métodos de Análise Envoltória de Dados (DEA), Programação matemática e variações (programação linear, inteira linear, inteira não linear, baseada em metas e multi objetivo), AHP e ANP, algoritmo genético, Lógica Fuzzy, Raciocínio baseado em casos (CBR) e SMART. Nas abordagens integradas destacam-se as técnicas associadas ao AHP (Bi negociação, DEA, redes neurais, grey relational analysis, programação não linear inteira mista, programação multi-objetivo) e aquelas que incorporam a lógica fuzzy em sua álgebra de cálculo matricial (AHP, análise de cluster, programação multiobjectivo, QFD, SMART, Algoritmo genético). Os autores classificam ainda em "outras abordagens combinadas" (Redes neurais e raciocínio baseado em casos; redes neurais e algoritmo genético; ANP e programação multiobjectivo; ANP e programação matemática por metas; DEA e programação multiobjectivo; DEA e SMART; e Algoritmo genético e programação multiobjectivo).

Na mesma linha de revisão de métodos de seleção de fornecedores, Chai, Liu e Ngai (2013) classificam-nos em três categorias: (a) métodos multicritério de tomada de decisão, (b) técnicas de programação matemática e (c) técnicas de inteligência artificial. O Quadro 1, a seguir, ilustra as categorias e os métodos que compõem a mesma. 
Quadro 1 - Técnicas de seleção de fornecedores

\begin{tabular}{|c|c|c|}
\hline Categoria & Técnica & Sigla \\
\hline \multirow{8}{*}{$\begin{array}{l}\text { Tomada de } \\
\text { decisão } \\
\text { multicritério }\end{array}$} & Analytic hierarchy process & $\mathrm{AHP}$ \\
\hline & Analytic network process & ANP \\
\hline & Elimination and Choice expressing reality & ELECTRE \\
\hline & Preference ranking organization method for enrichment evaluation & PROMETHEE \\
\hline & Tecnique for order performance by similatity to ideal solution & TOPSIS \\
\hline & Multicriteria optimization and compromisse solution & VIKOR \\
\hline & Decision making trial and evaluation laboratory & DEMATEL \\
\hline & Simple multiattribute rating technique & SMART \\
\hline \multirow{6}{*}{$\begin{array}{l}\text { Programação } \\
\text { matemática }\end{array}$} & Data envelopment analysis & DEA \\
\hline & Linear programming & LP \\
\hline & Non linear programming & NLP \\
\hline & Multiobjective programming & MOP \\
\hline & Goal programming & GP \\
\hline & Stochastic programming & SP \\
\hline \multirow{12}{*}{$\begin{array}{c}\text { Inteligência } \\
\text { Artificial }\end{array}$} & Genetic algorithm & GA \\
\hline & Grey system theory & GST \\
\hline & Neural networks & NN \\
\hline & Rough set theory & RST \\
\hline & Bayesian networks & $\mathrm{BN}$ \\
\hline & Decision tree & DT \\
\hline & Case-based reasoning & CBR \\
\hline & Particle swarm optimization & PSO \\
\hline & Support vector machine & SVM \\
\hline & Association rule & AR \\
\hline & Ant colony algorithm & ACA \\
\hline & Dempster shafer theory of evidence & DST \\
\hline
\end{tabular}

Fonte: Adaptado de Chai, Liu e Ngai (2013).

Os autores descrevem ainda o uso de combinado de técnicas ao AHP, ANP e DEA. Destaca-se uma significativa combinação de técnicas apropriadas para lidar com ambientes de incerteza, como a lógica fuzzy (e variações), por exemplo. Em menor expressão são descritas também abordagens integradas na categoria de métodos multicritério (ELECTRE, PROMETHEE e VIKOR) e na categoria Inteligência artificial (RST e GST).

O trabalho de Govindan et al. (2015) trata especificamente da descrição de técnicas usadas para apoiar o processo de seleção de fornecedores verdes. Seguindo a mesma lógica dos trabalhos apresentados anteriormente, os autores classificam as técnicas em abordagens individuais e combinada. Da primeira categoria destacam-se o AHP, o ANP e programação matemática. No caso do AHP, a incorporação da lógica fuzzy na álgebra do cálculo matricial do AHP não é considerada pelos autores como uma abordagem integrada. Destaca-se uma significativa quantidade de trabalhos no estilo Fuzzy-AHP (FAHP). A incorporação da lógica fuzzy no ANP para seleção de fornecedores verdes (FANP) ocorreu em menor grau que no caso do FAHP. Apenas um trabalho foi identificado pelos autores empregando programação matemática na seleção de fornecedores verdes. São classificadas ainda numa categoria "outras abordagens individuais" técnicas como DEA, Extensible synthetic model, técnicas estatísticas (testes de hipótese, análise 
fatorial, modelagem de equações estruturais), sistemas de inferência fuzzy, fuzzy TOPSIS, Choque integral, Grey entropy synthetic evaluation model, Knownledge based system (KBS), Rough set theory, Fuzzy multiagent decision making model e cross case analysis. Em relação ao uso combinado de técnicas destacam-se AHPNN, AHP-GA, NN e Multi Attribute Decision Analysis (MADA), DEA-ANP, DEAAHP/ANP, GST-AHP, Fuzzy set Theory e Grey Relational Analysis, KBS-CBR.

Os autores propõem ainda uma classificação de técnicas baseado no critério de seleção de fornecedores. A Tabela 1 descreve a frequência dos critérios mais citados nos artigos analisados pelos autores. Porém, não foi realizada uma análise do peso dos critérios.

Tabela 1 - Critérios mais citados nos artigos analisados

\begin{tabular}{lc}
\hline \multicolumn{1}{c}{ Critérios } & Freq. \\
\hline Environmental Management System & 11 \\
Quality & 8 \\
Service & 7 \\
Technology & 5 \\
Green Image & 4 \\
Design for environmental & 3 \\
Environmental competences & 3 \\
Environmental performance & 3 \\
Green competences & 3 \\
Price & 3 \\
Cost & 3 \\
\hline
\end{tabular}

Fonte: Proposto pelos autores a partir de Govindan et al. (2015).

Embora muitos estudos contemplem o uso de técnicas quantitativas para a seleção de fornecedores verdes, poucos trabalhos vêm incorporando a temática social nos modelos desenvolvidos (BAI; SARKIS, 2010a, 2010b).

Este trabalho explora também esta lacuna de pesquisa, ao apresentar uma revisão sistemática da literatura que amplia a proposta de Govidan et al. (2015), ao considerar também o termo sustentável (incluindo aspectos socais) nas pesquisas realizadas nas bases de dados.

\section{PROCEDIMENTOS METODOLÓGICOS}

Foi realizada uma revisão sistemática da literatura com o objetivo de descrever o estado da arte das metodologias/ferramentas utilizadas para a seleção de fornecedores sustentáveis. Como referência para a realização da revisão sistemática, foram adotados os procedimentos sugeridos por Cronin, Ryan e Coughlan (2008). O Quadro 2 ilustra os procedimentos adotados para a realização deste estudo.

Revista Produção Online, Florianópolis, SC, v. 16, n. 4, p. 1434-1457, out./dez. 2016. 
Quadro 2 - Procedimentos para a realização da revisão sistemática da literatura

\begin{tabular}{|c|c|}
\hline Parâmetros de busca & Descrição \\
\hline Bases de dados técnico científicas adotadas & $\begin{array}{l}\text { Scopus, Engineering Village (Compendex) e Web of } \\
\text { Science (Principal Coleção Web of Science) }\end{array}$ \\
\hline Filtro 1: tipo de material & artigos e artigos no prelo \\
\hline Filtro 2: termo de busca usados & 'select* green supplier' e 'select* sust* supplier' \\
\hline Filtro 3: Onde buscar os termos de busca do filtro 2 & Buscar apenas nos títulos dos artigos \\
\hline Filtro 4: delimitação do período da busca & De 2000 a 2015 \\
\hline Filtro 5: Verificar parâmetros de download do arquivo & $\begin{array}{l}\text { Baixar apenas artigos disponíveis na íntegra. Artigos } \\
\text { com apenas resumos foram descartados }\end{array}$ \\
\hline Filtro 6: Critério 1 de exclusão de um artigo & Eliminar artigos repetidos em outras bases \\
\hline Filtro 7: Critério 2 de exclusão de um artigo & $\begin{array}{l}\text { Artigos disponíveis apenas na forma de resumo ou } \\
\text { não gratuitos não eram procurados em bases como o } \\
\text { google scholar }\end{array}$ \\
\hline
\end{tabular}

A coleta nas bases de dados foi finalizada em dezembro de 2015. Após a execução dos passos, foram obtidos 27 artigos. Foram lidos apenas os resumos dos artigos, a fim de descrever os principais conceitos a eles relacionados, a saber: ano de publicação, palavras-chave, periódico em que foi publicado, método ou ferramenta multicritério utilizado, entre outras características. Para mensuração da revisão sistemática da literatura realizada, foram utilizadas estatísticas descritivas, análise de conteúdo dos títulos e palavras-chave via tag cloud e a análise de correspondência. Foram desconsiderados os artigos de revisões sistemáticas da literatura e também trabalhos que não consideravam como objeto de estudo a aplicação de métodos voltados à seleção de fornecedores.

Para classificação das técnicas, foi utilizada como referências os trabalhos de Ho, Xu e Dey (2010), Chai, Liu e Ngai (2013) e Govidan et al. (2015). A Figura 2 ilustra o modelo de classificação de técnicas de seleção de fornecedores utilizado nesta pesquisa.

As técnicas utilizadas para a seleção de fornecedores podem ser agrupadas em dois grandes grupos, a saber: abordagem individual, e abordagem combinada. A abordagem individual compreende o emprego de uma única técnica para a etapa de seleção de fornecedores. Nesse contexto, constata-se o uso de técnicas multicritério de tomada de decisão (MCDM), técnicas de programação matemática (PM), técnicas baseadas em inteligência artificial (IA) e outras técnicas diversas, como por exemplo, Entropy method, System dynamics, Axiomatic Design, Composite indicators method, Bayesian statistics, Linear assignment method, Spearman rank correlation coeficiente, entre outros. 
Figura 2 - Classificação das técnicas que suportam a seleção de fornecedores verdes/sustentáveis

\section{Seleção de fornecedores verdes/sustentáveis}

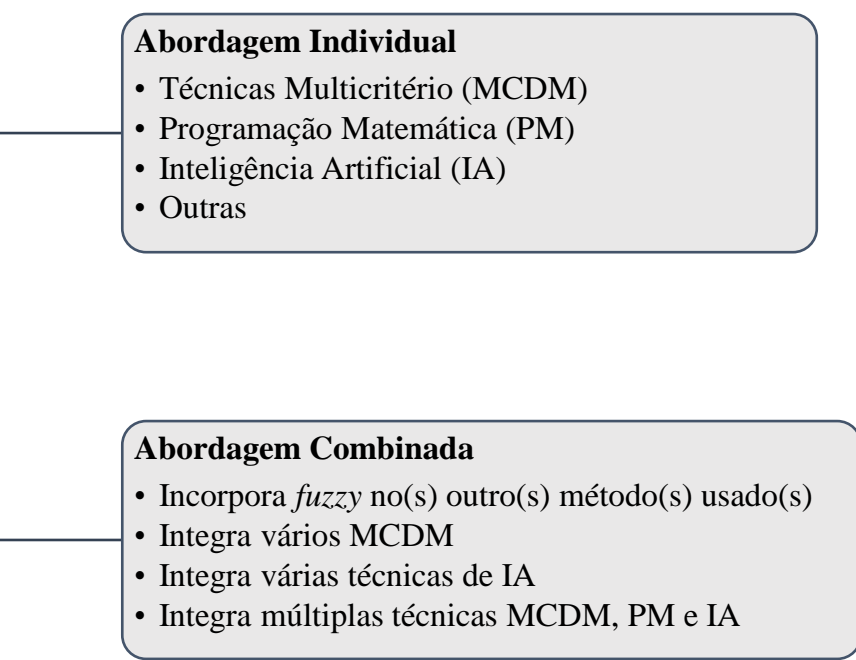

Fonte: Proposto pelos autores.

A abordagem combinada, por sua vez, congrega a utilização de duas ou mais técnicas aplicadas de forma integrada para a seleção de fornecedores verdes/sustentáveis. Nesse sentido, o uso combinado foi dividido em quatro categoria de uso múltiplo de técnicas: (a) técnicas que incorporam a lógica fuzzy na álgebra de cálculo do método considerado; (b) uso combinado de várias técnicas MCDM; (c) uso combinado de técnicas de inteligência artificial; e (d) uso de múltiplas técnicas dentre MCDM, PM, IA e "outras técnicas".

\section{APRESENTAÇÃO E DISCUSSÃO DOS RESULTADOS}

Em relação à quantidade de artigos publicados, verificou-se que somente a partir de 2009 foram publicados os primeiros artigos relacionando seleção de fornecedores à temática ambiental ou sustentável. No entanto, a partir de 2014 o crescimento aumentou significativamente, conforme ilustrado na Figura 3. 
Figura 3 - Frequência de artigos publicados

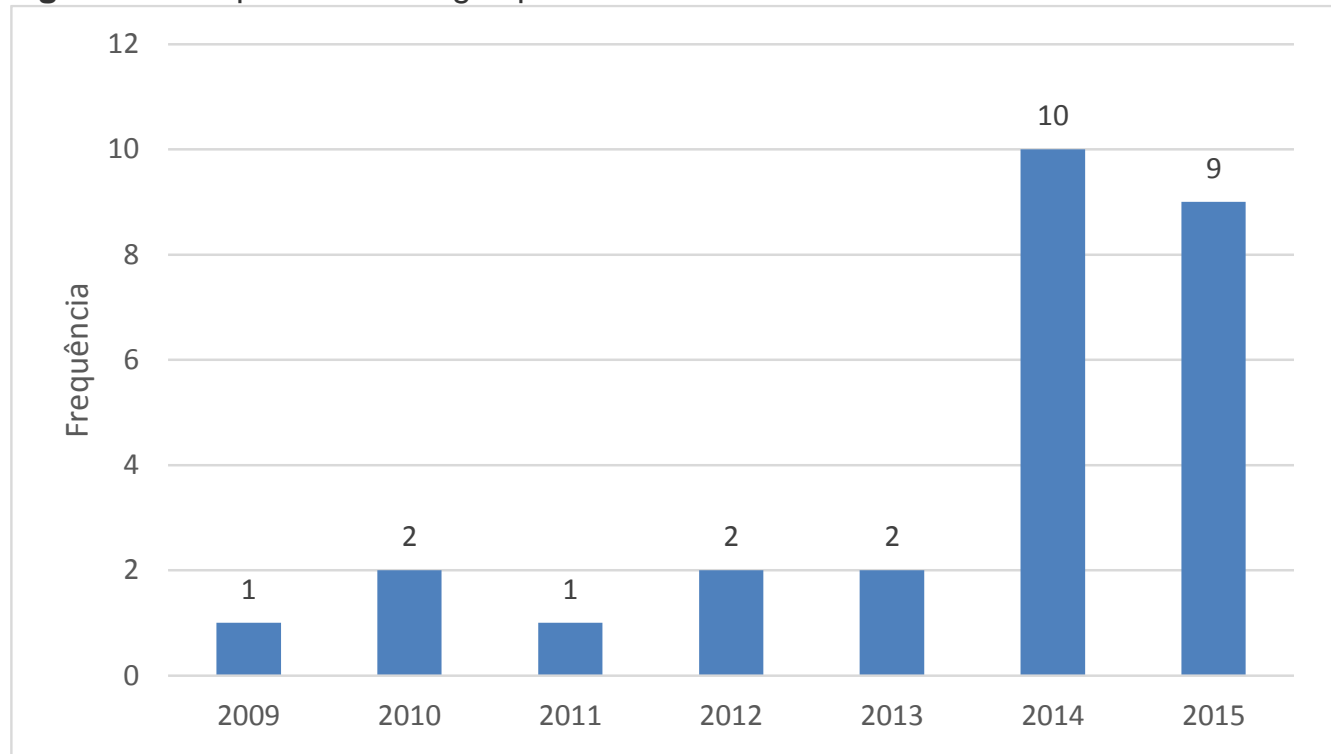

Fonte: Dados da Pesquisa

Em relação ao foco do modelo de seleção (se fornecedor verde ou sustentável), verificou-se que a maior parte dos artigos (63\%) focavam a seleção de fornecedores verdes, enquanto que uma menor parcela $(37 \%)$ dos modelos incorporaram uma perspectiva mais ampla (sustentável). Os periódicos que mais publicaram sobre o tema foram Journal of Cleaner Production, com 5 artigos, o International Journal of Production Economics, com 4 artigos, o Sustainability, com 3 artigos e o Mathematical Problems in Engineering com 2 artigos publicados, perfazendo mais de $50 \%$ dos artigos publicados.

Em relação ao método de classificação das técnicas (Figura 2), a maior parte (85\%) dos artigos (KHAMSEH; MAHMOODI, 2014; BAI; SARKIS, 2010; BAKESHLOU et al., 2014; BÜYÜKÖZKAN; ÇIFÇI, 2011; CAO; WU; LIANG, 2015; DOBOS; VÖRÖSMARTY, 2014; FREEMAN; CHEN, 2015; HASHEMI; KARIMI; TAVANA, 2015; JIA et al., 2015; KANNAN et al., 2013; KANNAN; SOUSA JABBOUR; JABBOUR, 2014; KANNAN; GOVINDAN; RAJENDRAN, 2014; KUO; HSU; LI, 2015; KUO; WANG; TIEN, 2010; LEE et al., 2009; ORJI; WEI, 2014; ORJI; WEI, 2015; SARKIS; DHAVELE, 2015; TAN et al., 2014; TSUI; WEN, 2014; YAZDANI, 2014; ZHAO; GUO, 2014; ZHOU et al., 2012) aplicou de forma combinada as técnicas para seleção de fornecedores verdes/sustentáveis.

Somente 4 artigos (15\%) utilizaram uma técnica de forma individual para a seleção de fornecedores verdes/sustentáveis. As quatro técnicas aplicadas de forma individual foram: o uso de um sistema de inferência fuzzy (IA) (AMINDOUST et al., 2012), a programação inteira (PM) (TRAPP; SARKIS, 2015), a Análise Envoltória de Dados (PM)(SHI et al., 2014) e DEMATEL (MCDM) (HSU et al., 2013). Desta forma, o uso individual ficou caracterizado pelo maior uso de técnicas relacionadas a programação matemática. A Tabela 2, por sua vez descreve o uso de técnicas combinadas para a seleção de fornecedores. 
Tabela 2 - Uso combinado de técnicas

\begin{tabular}{lcc}
\hline Uso combinado de técnicas & Freq. & $\%$ \\
\hline Múltiplas combinações entre as categorias & 13 & $57 \%$ \\
Incorpora lógica fuzzy & 7 & $30 \%$ \\
MCDM & 2 & $9 \%$ \\
IA & 1 & $4 \%$ \\
\hline \multicolumn{2}{r}{} \\
\hline
\end{tabular}

A maior parte $(57 \%)$ dos artigos que combinam técnicas de seleção de fornecedores verdes/sustentáveis o fazem por meio de técnicas variadas entre as categorias descritas na Figura 2, a saber: MCDM, IA, PM, e "outras técnicas". A possibilidade de combinações entre as categorias de técnicas é ampla. No entanto, destaca-se a combinação de técnicas MCDM com "outras técnicas" (MCDM \& Outras técnicas), e também a possibilidade de incorporação da lógica fuzzy nas técnicas MCDM integradas as "outras técnicas" (Fuzzy MCDM \& "Outras técnicas"). Observa-se também a combinação de técnicas MCDM \& IA. Um fato de destaque é que $85 \%$ dos artigos que promoveram o uso de técnicas diversas, apresentavam sempre uma técnica MCDM integrada a "outra técnica" ou ainda a incorporação da lógica fuzzy à técnica MCDM.

Ainda discutindo a aplicação de múltiplas técnicas na seleção de fornecedores, destaca-se a incorporação da lógica fuzzy nos variados métodos de seleção de fornecedores em 7 artigos (30\% dos artigos que combinam várias categorias de técnicas). Deste total, 5 artigos (70\%) representam a incorporação da lógica fuzzy em métodos MCDM. Os métodos MCDM que mais incorporam a lógica fuzzy em sua algebra de cálculo foram AHP, o ANP e o TOPSIS. A combinação de técnicas do tipo "MCDM \& MCDM" e "IA \& IA" ocorreram em menor grau (13\%).

A Figura 4 mostra uma análise de conteúdo das palavras contidas no título dos artigos analisados. O tamanho das palavras é proporcional à quantidade de vezes que as mesmas são declaradas (tag cloud, ou word cloud). 
Figura 4 Tag cloud para palavras contidas nos títulos dos artigos

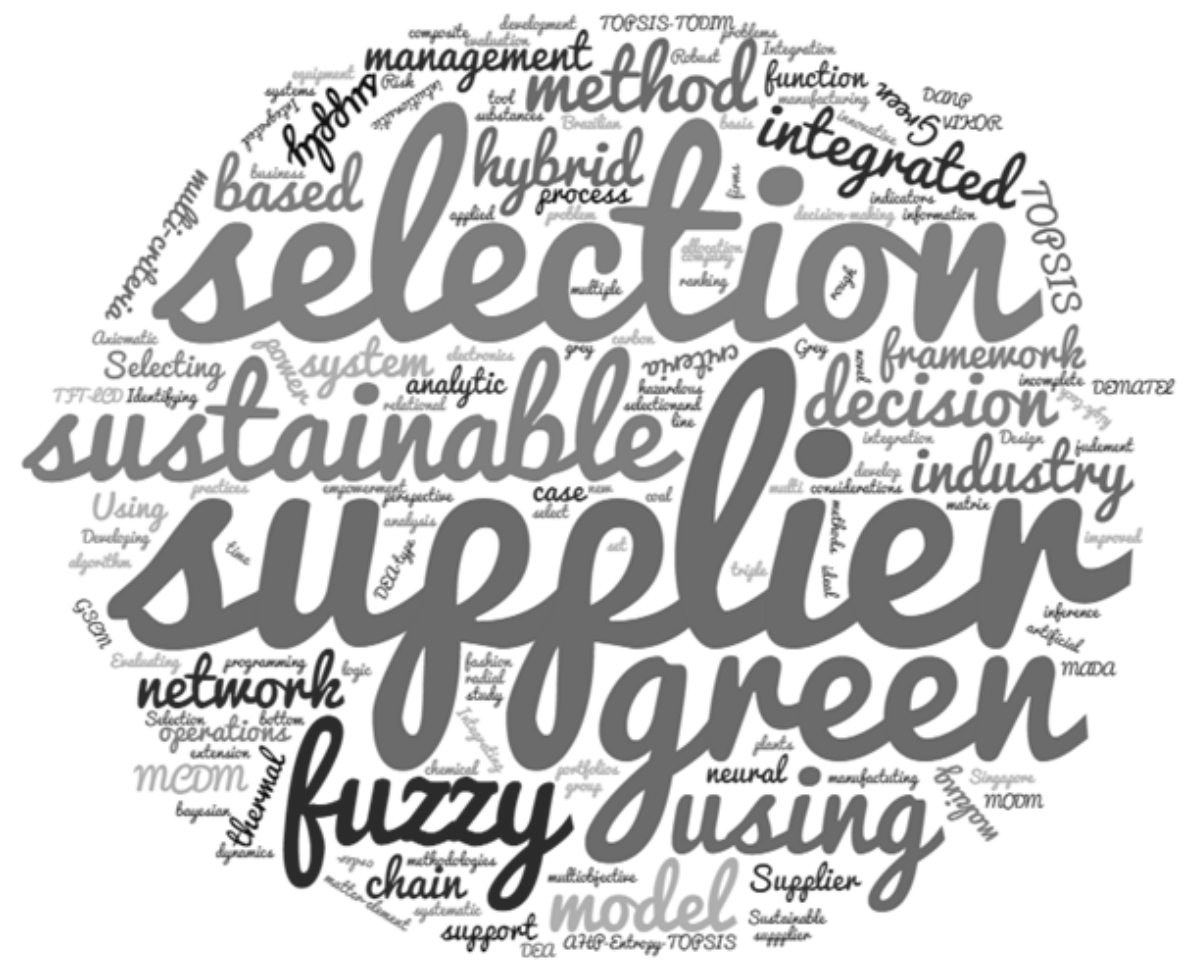

Fonte: Dados da Pesquisa

Observa-se que há uma predominância das palavras "Supplier", "Selection", "Green", "Sustainable" e "fuzzy". Isto nos leva a concluir que a maior parte dos artigos sobre seleção de fornecedores focam predominantemente a questão ambiental, denotada pelo termo "green" ou "verde", corroborando a evidência de Bai e Sarkis (2010a, 2010b). Além disso, há um destaque para a incorporação da lógica fuzzy nos diversos métodos de seleção de fornecedores.

É importante ressaltar que existe uma lacuna na literatura para trabalhos que desenvolvam métodos de seleção de fornecedores a partir de uma perspectiva mais ampla, ou seja, considerando além da questão ambiental, a social. Essa visão mais abrangente é denotada pelo termo "sustentável".

Uma análise tag cloud das palavras-chave (Figura 5) contidas nos artigos reforça a evidência obtida na análise do titulo dos artigos (Figura 4). 
Figura 5 Tag cloud das palavras-chave usadas nos artigos

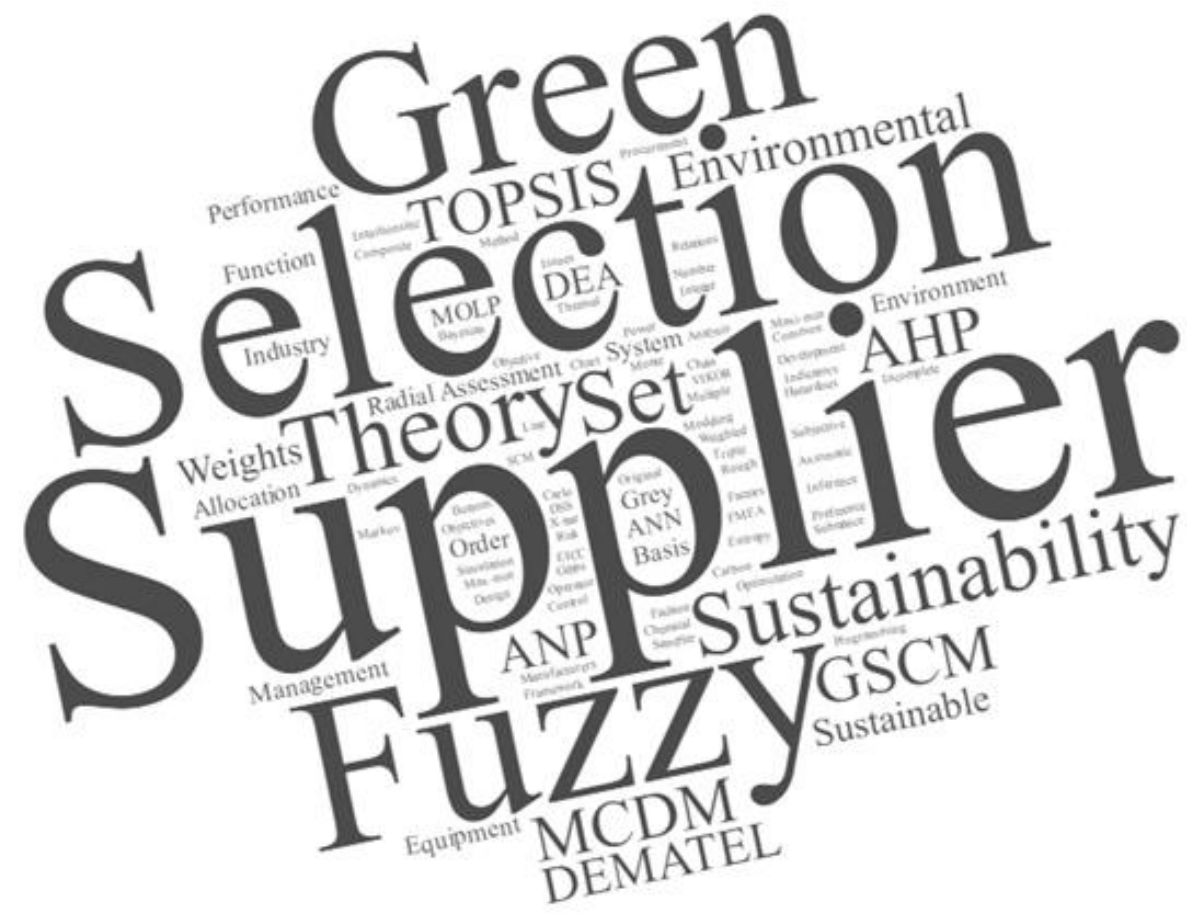

Fonte: Dados da Pesquisa.

Ainda analisando as palavras-chave contidas nos artigos, porém considerando somente termos relativos às técnicas de seleção de formadores, destaca-se novamente a lógica Fuzzy e os Métodos MCDM, reforçando a análise descritiva destacada anteriormente, como pode ser observado na Figura 6 a seguir.

Figura 6 Tag cloud das palavras-chave considerando apenas as técnicas de seleção de fornecedores

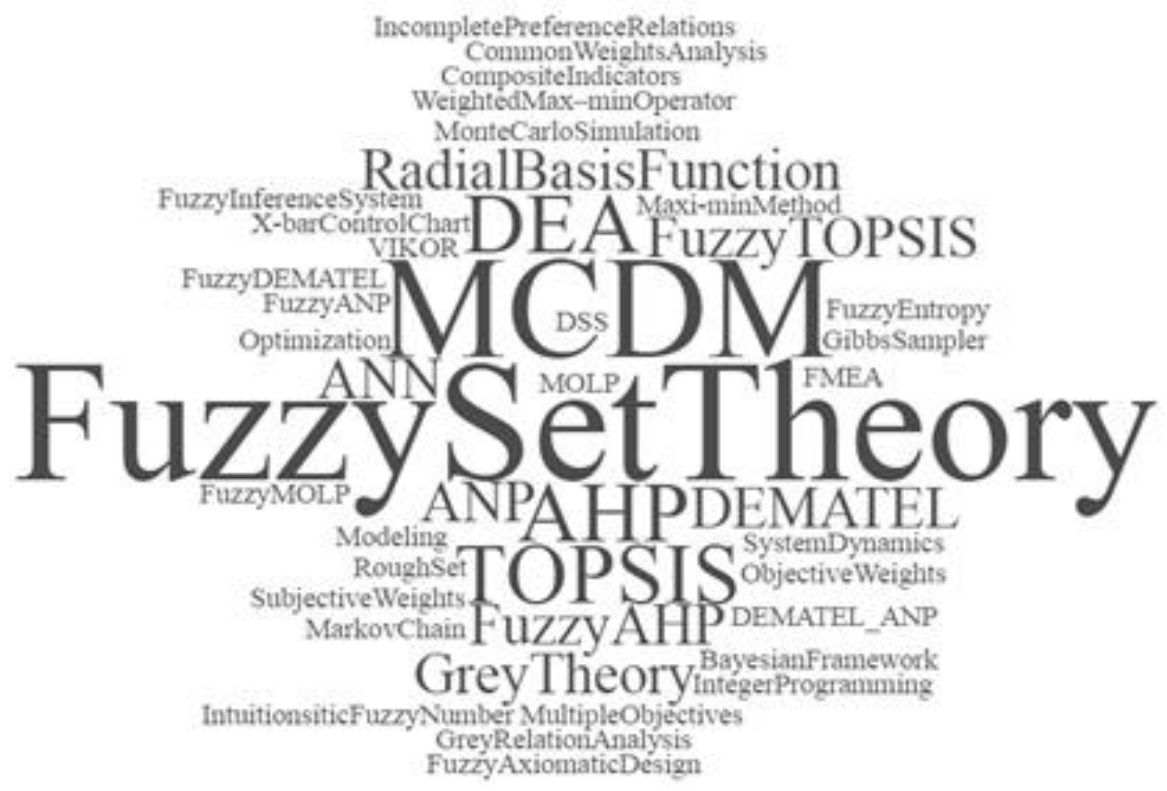

Fonte: Dados da Pesquisa

Revista Produção Online, Florianópolis, SC, v. 16, n. 4, p. 1434-1457, out./dez. 2016. 
A análise de correspondência (ANACOR) (WHITLARK; SMITH, 2001) é uma técnica que exibe associações entre um conjunto de variáveis categóricas nominais em um mapa perceptual, permitindo, deste modo, uma análise visual de qualquer padrão de utilização de métodos e ferramentas utilizados na seleção de fornecedores verdes. Neste trabalho, foi utilizada a projeção simétrica (GREENACRE, 2007), que permite explorar simultaneamente as relações entre linhas e colunas dos quadros de contingência, ou seja, as associações entre todas as categorias de ambas as variáveis. Isso equivale dizer que categorias com localização próxima na projeção plana têm relação mais forte do que categorias separadas por distâncias maiores.

Diante da evidência do uso combinado de técnicas na seleção dos fornecedores, foi realizada uma análise de correspondência para verificar possíveis padrões de relação entre o foco da seleção (verde ou sustentável) e as técnicas combinadas usadas. A Figura 7 ilustra tal relação.

Figura 7 - Mapa perceptual relacionando foco da seleção de fornecedores (verde ou sustentável) e uso combinado de técnicas

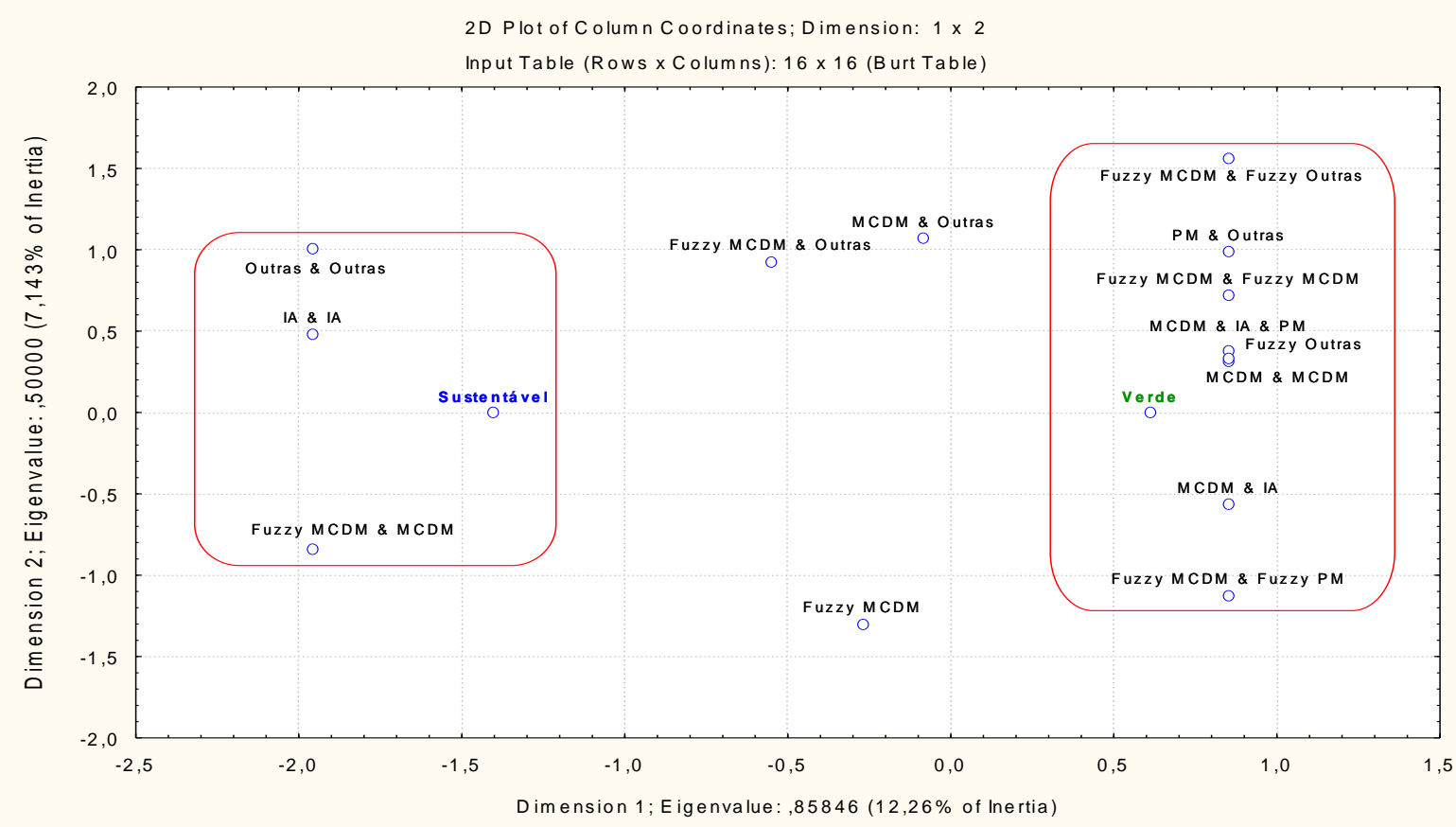

Fonte: Dados da Pesquisa

Observando o lado direito do mapa perceptual, relativo à seleção de fornecedores verdes, destaca-se o uso intensivo de métodos MCDM combinado à outras técnicas, dentre elas técnicas MCDM, e também a incorporação da lógica fuzzy nos/entre métodos MCDM. Além desse fato, percebe-se a utilização da Programação Matemática \& "Outras técnicas", e também da incorporação da lógica fuzzy nas "Outras técnicas". Quando o foco envolve a seleção de fornecedores 
sustentáveis, observa-se novamente a presença combinada de técnicas MCDM (MCDM \& MCDM), IA (IA \& IA) e "Outras \& Outras".

Em menor grau de utilização, tanto para a seleção de fornecedores verdes quanto sustentáveis, registra-se a incorporação da lógica fuzzy nas técnicas MCDM (Fuzzy AHP, Fuzzy ANP e Fuzzy TOPSIS, por exemplo) e combinadas às "outras técnicas" (Fuzzy MCDM \& Outras); o uso combinado de técnicas MCDM à outras técnicas (MCDM \& Outras); e a incorporação da lógica fuzzy nas técnicas MCDM (Fuzzy MCDM).

\section{CONSIDERAÇÕES FINAIS}

A seleção de fornecedores é uma atividade essencial na gestão da cadeia de suprimentos. O aumento da consciência ambiental e social pelos clientes (sejam consumidores finais ou clientes corporativos no contexto do B2B) vêm estimulando as empresas à considerarem estes critérios na seleção de fornecedores.

A variedade de critérios e as respectivas naturezas desses constructos e variáveis, tornam-se cada vez mais complexas e subjetivas. Desta forma é necessário que as técnicas de seleção de fornecedores encapsulem as restrições de avaliações qualitativas e quantitativas para selecionar de forma adequada os parceiros de negócios.

Desta forma, compreender as técnicas e as condições de uso e contexto em que foram aplicadas para selecionar fornecedores verdes/sustentáveis é fundamental. Para tanto, este artigo empreendeu uma revisão sistemática da literatura a fim de mapear o uso de técnicas e ferramentas que vêm sendo utilizadas na seleção de fornecedores.

As principais evidências encontradas na literatura sobre seleção de fornecedores verdes foi o uso intensivo de técnicas associadas em detrimento da utilização da técnica de forma única. Outra evidência, já ressaltada na literatura clássica sobre métodos de seleção de fornecedores, é a extensiva incorporação da lógica fuzzy nos métodos usuais, como os MCDM, os PM e até IA.

Percebeu-se, também, uma lacuna que vai além da exploração de técnicas de seleção de fornecedores : o emprego de uma visão mais holística na seleção de fornecedores verdes/sustentáveis e sua relação com demais processos de negócio de abastecimento e gestão de cadeia, bem como com os agentes ao longo da cadeia imediata. Neste sentido, abordagens qualitativas, como por exemplo, o estudo de caso e pesquisas de levantamento seriam interessantes para exploração dos constructos envolvidos na seleção de fornecedores verdes/sustentáveis.

Um destaque positivo deste trabalho foi o emprego da técnica de cloud words para análise de conteúdo dos trabalhos e também da análise de correspondência. Não é comum encontrar revisões sistemáticas de literatura que empreguem tais métodos no processo de descrição e análise de conteúdo dos artigos. Tais 
ferramentas constituem uma oportunidade maior de exploração e entendimento de uma revisão sistemática de literatura.

Ainda, cabe ressaltar que a constatação de uma menor taxa de utilização de técnicas na seleção de fornecedores sustentáveis (não somente verdes) não significa que não seja pertinente aplicar a variedade de técnicas e respectivas combinações encontradas na seleção de fornecedores verdes. Possivelmente, essa baixa amplitude de variedade de técnicas e combinações possíveis se deva à incorporação mais recente da perspectiva sustentável.

Importante se faz ressaltar que há uma demanda latente na literatura por explorar a aplicação de técnicas (e suas possíveis combinações) na seleção de fornecedores sustentáveis que incorporem o lado social de forma explícita e profunda. Esta pesquisa encerra sua análise por meio de uma proposição a ser testada e validade em trabalhos futuros sobre a seleção de fornecedores verdes/sustentáveis:

Proposição final da pesquisa: A utilização combinada de abordagens/técnicas/ferramentas, permite uma melhor compreensão e tratamento da problemática de seleção de fornecedores sustentáveis do que a utilização de uma técnica de forma única/isolada.

Esta proposição demanda, dessa forma, estudos que combinem diferentes técnicas em diferentes contextos e aplicação a fim de verificar pontos positivos e negativos do uso combinado das mesmas.

\section{REFERÊNCIAS}

AMINDOUST, A.; AHMED, S.; SAGHAFINIA, A.; BAHREININEJAD, A. Sustainable supplier selection: A ranking model based on fuzzy inference system. Applied Soft Computing Journal, v. 12, n. 6, p. 1668-1677, 2012. DOI:10.1016/j.asoc.2012.01.023

BAI, C.; SARKIS, J. Green supplier development: analytical evaluation using rough set theory. International Journal of Cleaner Production, v.18, p. 1200-1210, 2010a. DOI:10.1016/j.jclepro.2010.01.016

BAI, C.; SARKIS, J. Integrationg sustainability into supplier selection with Grey system and rought set metodologies. International Journal of Production Economics., v.124, p. 252264, 2010b. DOI:10.1016/j.ijpe.2009.11.023

BAKESHLOU, E. A.; KHAMSEH, A. A.; ASL, M. A. G.; SADEGHI, J.; ABBASZADEH, M. Evaluating a green supplier selection problem using a hybrid MODM algorithm. Journal of Intelligent Manufacturing, [2014]. (In press) DOI: 10.1007/s10845-014-1028-y

BÜYÜKÖZKAN, G.; ÇIFÇI, G. A novel fuzzy multi-criteria decision framework for sustainable supplier selection with incomplete information. Computers in Industry, v. 62, n. 2, p. 164174, 2011. DOI:10.1016/i.compind.2010.10.009 
CARTER, C.R.; EASTON, P.L. Sustainable supply chain management: Evolution and future directions. International Journal of Physical Distribution and Logistics Management, v. 41, n. 1, p. 46-62, 2011. DOI: 10.1108/09600031111101420

CHAI, J.; LIU, J.N.K.; NGAI, E.W.T. Application of decision-making techniques in supplier selection: A systematic review of literature. Expert Systems with Applications, v. 40, n. 10, p. 3872-3885, 2013. DOI: 10.1016/j.eswa.2012.12.040

CAO, Q.; WU, J.; LIANG, C. An intuitionsitic fuzzy judgement matrix and TOPSIS integrated multi-criteria decision making method for green supplier selection. Journal of Intelligent \& Fuzzy Systems, vol. 28, no. 1, pp. 117-126, 2015. DOI: 10.3233/IFS-141281

COUSINS, P.; LAMMING, R.; LAWSON, B.; SQUIRE, B. Strategic Supply Management: principles, theories and practice. Harlow: Prentice Hall, 2008.

CRONIN, P.; RYAN, F.; COUGHLAN, M. Undertaking a literature review: a step-by-step approach. The British journal of nursing, v. 17, n. 1, p. 38-43, 2008. DOI :

$\underline{10.1177 / 107808747000500401}$

De BOER, L.; LABRO, E.; MORLACHI, P. A review of methods supporting supplier selection. European Journal of Purchasing \& Supply Management, v.7, p. 75-89, 2001. DOI: $10.1016 /$ S0969-7012(00)00028-9

DE BOER, L.; WEGEN, L. V. D.; TELGEN,J. Outranking methods in support of supplier selection. European Journal of Purchasing \& Supply Management, v. 4, p. 109-118, 1998. DOI: $\underline{10.1016 / S 0969-7012(97) 00034-8}$

DOBOS, I.; VÖRÖSMARTY, G. Green supplier selection and evaluation using DEA-type. Intern. Journal of Production Economics, v. 157, p. 273-278, 2014.

DOI:10.1016/j.ijpe.2014.09.026

ELKINGTON, J. Cannibals with Forks: The Triple Bottom Line of 21st Century Business. New Society Publishers, 1998. DOI: 10.1023/A:1006129603978

FREEMAN, J.; CHEN, T. Green supplier selection using an AHP-Entropy-TOPSIS framework. Supply Chain Management: An International Journal, v. 20, n. 3, p.327- 340, 2015. DOI:10.1108/SCM-04-2014-0142.

GANGA, G. M. D. Trabalho de Conclusão de Curso (TCC) na Engenharia de Produção: um guia prático de conteúdo e forma. São Paulo: Atlas, 2012.

GOVINDAN, K.; RAJENDRAN, S.; SARKIS, J.; MURUGESAN, P. Multi criteria decision making approaches for green supplier evaluation and selection: A literature review. Journal of Cleaner Production, v. 98, p. 66-83, 2015. DOI: 10.1016/.j.jclepro.2013.06.046.

GREENACRE, M. Correspondence Analysis in practice. 2 ed. New York: Chapman and Hall, 2007. ISBN 9781584886167.

HACKING, T.; GUTHRIE, P. A framework for clarifying the meaning of Triple Bottom-Line, Integrated, and Sustainability Assessment. Environmental Impact Assessment Review, v. 28, n. 2-3, p. 73-89, 2008. DOI: 10.1016/j.eiar.2007.03.002 
HASHEMI, S. H.; KARIMI, A.; TAVANA, M. An integrated green supplier selection approach with analytic network process and improved Grey relational analysis. Intern. Journal of Production Economics, v. 159, p. 178-191, 2014. DOI:10.1016/j.ijpe.2014.09.027.

HERVANI, A.A.; HELMS, M.M.; SARKIS, J. Performance measurement for green supply chain management. Benchmarking, v. 12, n. 4, p. 330-353, 2005. DOI:

$\underline{10.1108 / 14635770510609015 .}$

HO, W.; XU, X.; DEY, P.K. Multi-criteria decision-making approaches for supplier evaluation and selection: A literature review. European Journal of Operational Research, v. 202, n. 1, p. 16-24, 2010. DOI: 10.1016/j.ejor.2009.05.009.

HSU, C.-W.; KUO, T.-C.; CHEN, S.-H.; HU, A. H. Using DEMATEL to develop a carbon management model of supplier selection in green supply chain management. Journal of Cleaner Production, v. 56, n. 1, p. 164-172, 2013. DOI:10.1016/j.jclepro.2011.09.012.

HUBBARD, G. Measuring Organizational Performance: Beyond the Triple Bottom Line. Business Strategy and the Environment, v. 19, p.177-191, 2009. DOI: $10.1002 /$ bse.564

IGARASHI, M.; DE BOER, L.; FET, A.M. What is required for greener supplier selection? A literature review and conceptual model development. Journal of Purchasing and Supply Management, v. 19, n. 4, p. 1-17, 2013. DOI: 10.1016/i.pursup.2013.06.001.

ITTNER, C.D.; LARCKER, D.F.; NAGAR, V.; RAJAN, M.V. Supplier selection, monitoring practices, and firm performance. Journal of Accounting and Public Policy, v. 18, n. 3, p. 253-281, 1999. DOI:10.1016/S0278-4254(99)00003-4.

JABBOUR, A. B. L. S.; JABBOUR, C. J. C. Are supplier selection criteria going green? Case studies of companies in Brazil. Industrial Management \& Data Systems, v. 109, p. 477495, 2009. DOI: $\underline{10.1108 / 02635570910948623}$

JIA, P.; GOVINDAN, K.; CHOI, T.-M.; RAJENDRAN, S. Supplier Selection Problems in Fashion Business Operations with Sustainability Considerations. Sustainability, v. 7, n. 2, p. 1603-1619, 2015. DOI:10.3390/su7021603.

KANNAN, D.; GOVINDAN, K.; RAJENDRAN, S. Fuzzy Axiomatic Design approach based green supplier selection: a case study from Singapore. Journal of Cleaner Production, p.115, 2014. DOI: /10.1016/j.jclepro.2013.12.076.

KANNAN, D.; KHODAVERDI, R.; OLFAT, L.; JAFARIAN, A.; DIABAT, A. Integrated fuzzy multi criteria decision making method and multiobjective programming approach for supplier selection and order allocation in a green supply chain. Journal of Cleaner Production, v. 47, p. 355-367, 2013. DOI:10.1016/.j.jclepro.2013.02.010.

KANNAN, D.; SOUSA JABBOUR, A. B. L. DE; JABBOUR, C. J. C. Selecting green suppliers based on GSCM practices: Using Fuzzy TOPSIS applied to a Brazilian electronics company. European Journal of Operational Research, v. 233, n. 2, p. 432-447, 2014. DOI:10.1016/j.ejor.2013.07.023.

KANNAN, V.R.; TAN, K.C. Buyer-supplier relationships: The impact of supplier selection and buyer-supplier engagement on relationship and firm performance. International Journal of 
Physical Distribution and Logistics Management, v. 36, n. 10, p. 755-775, 2006. DOI: $\underline{10.1108 / 09600030610714580 .}$

KHAMSEH, A. A.; MAHMOODI, M. A New Fuzzy TOPSIS-TODIM Hybrid Method for Green Supplier Selection Using Fuzzy Time Function. Advances in Fuzzy Systems, v. 2014, 2014. DOI: $10.1155 / 2014 / 841405$.

KUO, R. J.; WANG, Y. C.; TIEN, F. C. Integration of artificial neural network and MADA methods for green supplier selection. Journal of Cleaner Production, v. 18, n. 12, p. 11611170, 2010. DOI:10.1016/j.jclepro.2010.03.020.

KUO, T.; HSU, C.-W.; LI, J.-Y. Developing a Green Supplier Selection Model by Using the DANP with VIKOR. Sustainability, v. 7, p. 1661-1689, 2015. DOI:10.3390/su7021661.

LEE, A. H. I.; KANG, H.-Y.; HSU, C.-F.; HUNG, H.-C. A green supplier selection model for high-tech industry. Expert Systems with Applications, v. 36, n. 4, p. 7917-7927, 2009. DOI:10.1016/i.eswa.2008.11.052.

LIN, C.-T.; CHEN, C.-B.; TING, Y.-C. A green purchasing model using ANP and LP methods. Journal of Testing and Evaluation, v. 40, n.2, p. 203-210, 2012. DOI: 10.1520/JTE104259.

MIN, H.; GALLE, W. P. Green purchasing practices of US firms", International Journal of Operations \& Production Management, v. 21, n. 9, p.1222 - 1238, 2001. DOI: 10.1108/EUM0000000005923.

NAIR, A.; JAYARAM, J.; DAS, A. Strategic purchasing participation, supplier selection, supplier evaluation and purchasing performance. International Journal of Production Research, v. 53, n. 20, p. 6263-6278, 2015. DOI: 10.1080/00207543.2015.1047983

NORMAN, W.; MACDONALD, C. Getting to the Bottom of "Triple Bottom Line". Business Ethics Quarterly, v. 14, n. 2, 2004, p. 243-262, 2004. DOI:

http://dx.doi.org/10.5840/beq200414211

ORJI, I. J.; WEI, S. A Decision Support Tool for Sustainable Supplier Selection in Manufacturing Firms. Journal of Industrial Engineering and Management, v. 7, n. 5, p. 1293-1315, 2014. DOI: 10.3926/jiem.1203.

ORJI, I. J.; WEI, S. An innovative integration of fuzzy-logic and systems dynamics in sustainable supplier selection: A case on manufacturing industry. Computers \& Industrial Engineering, v. 88, p. 1-12, 2015. DOI:10.1016/i.cie.2015.06.019.

RIEBEL, P. "Greening" the supply chain. Pulp \& Paper-Canada, v. 100, n. 9 p. 63-63, 1999. DOI nao disponível.

SALAMIN, X.; HANAPPI, D. Women and international assignments: A systematic literature review exploring textual data by correspondence analysis. Journal of Global Mobility, v. 2, n. 3, p. 343-374, 2014. DOI: 10.1108/JGM-09-2013-0058.

SARKIS, J. A strategic decision framework for green supply chain management. Journal of Cleaner Production, v. 11, n. 4, p. 397-409, 2003. DOI: 10.1016/S0959-6526(02)00062-8. 
SARKIS, J.; DHAVALE, D. G. Supplier selection for sustainable operations: A triple-bottomline approach using a Bayesian framework. International Journal of Production Economics, v.166, p.177-191, 2015. DOI:10.1016/j.ijpe.2014.11.007.

SELLITO, M. A.; BORCHARDT, M.; PEREIRA, G. M.; PACHECO, D. A. de J. Gestão de cadeias de suprimentos verdes: quadro de trabalho. Produção Online, Florianópolis, SC, v. 13, n.1, p. 351-374, jan./mar. 2013. DOI: http://dx.doi.org/10.14488/1676-1901.v13i1.1181.

SEURING, S.; MÜLLER, M. From a literature review to a conceptual framework for sustainable supply chain management. Journal of Cleaner Production, v. 16, n. 15, p. 1699-1710, 2008. DOI: 10.1016/j.jclepro.2008.04.020.

SHI, P.; YAN, B.; SHI, S.; KE, C. A decision support system to select suppliers for a sustainable supply chain based on a systematic DEA approach. Information Technology and Management, v. 16, n. 1, p. 39-49, 2014. DOI 10.1007/s10799-014-0193-1.

SINCLAIR, J.; CARDEW-HALL, M. The folksonomy tag cloud: When is it useful? Journal of Information Science, v. 34, n. 1, p. 15-29, 2008. DOI: 10.1177/0165551506078083.

SMITH, B. Global environmental trends: greening of the textile supply chain. American Dyestuff Reporter, v. 87, n. 9, Sep. 1998.

SRIVASTAVA, S.K. Green supply-chain management: A state-of-the-art literature review. International Journal of Management Reviews, v. 9, n. 1, p. 53-80, 2007. DOI: 10.1111/j.1468-2370.2007.00202.x.

STEVENSON, F.; JONES, M.; MACRAE, J. Mapping building materials and products in remote areas: Sustainable supply chains and the specifier. Built Environment, v. 28, n.1, p. 33-45, 2002. DOI: $10.2307 / 23288549$.

TAN, Z.; JU, L.; YU, X.; ZHANG, H.; YU, C. Selection Ideal Coal Suppliers of Thermal Power Plants Using the Matter-Element Extension Model with Integrated Empowerment Method for Sustainability. Mathematical Problems in Engineering , v. 2014, p. 1-11, 2014. DOI: 10.1155/2014/302748.

TRACEY, M.; TAN, C.L. Empirical analysis of supplier selection and involvement, customer satisfaction, and firm performance. Supply Chain Management, v. 6, n. 4, p. 174-188, 2001. DOI: 10.1108/EUM0000000005709.

TRAPP, A. C.; SARKIS, J. Identifying Robust portfolios of suppliers: a sustainability selection and development perspective. Journal of Cleaner Production, p. 1-13, [2014?]. (In press). DOI:10.1016/j.jclepro.2014.09.062.

TSUI, C.; WEN, U. A Hybrid Multiple Criteria Group Decision-Making Approach for Green Supplier Selection in the TFT-LCD Industry. Mathematical Problems in Engineering, 2014. DOI: $\underline{10.1155 / 2014 / 709872 .}$

VAN HOEK, R. I. From reversed logistics to green supply chains. Supply Chain Management, v. 4, n. 3, p. 129-134, 1999. DOI: $10.1108 / 13598549910279576$.

VAN WEELE, A. J. Purchasing \& Supply Chain Management: analysis, strategy, planning and practice. Andover: Cengage Learning, 2010. DOI: 10.4324/9781315819211. 
VISWANADHAM, N.; SAMVEDI, A. Supplier selection based on supply chain ecosystem, performance and risk criteria. International Journal of Production Research, v. 51, n. 21, p. 6484-6498, 2013. DOI: $\underline{10.1080 / 00207543.2013 .825056 .}$

WEBB, L. Green purchasing: forging a new link in the supply chain. Resource: Engineering and Technology for Sustainable World, 1 (6), pp. 14-18, 1994.

WHITLARK, D. B.; SMITH, S. M. Using correspondence analysis to map relationships. Marketing Research, v. 13, n. 3, p.22-27, 2001.

YAZDANI, M. An integrated MCDM approach to green supplier selection. International Journal of Industrial Engineering Computations, v. 5, p. 443-458, 2014. DOI: 10.5267/j.ijiec.2014.3.003

ZHANG, D.W.; ABDUL HAMID, A.B.; THOO, A.C. Sustainable supplier selection: An international comparative literature review for future investigation. Applied Mechanics and Materials, v. 525, p. 787-790, 2014. DOI: 10.4028/www.scientific.net/AMM.525.787

ZHAO, H.; GUO, S. Selecting green supplier of thermal power equipment by using a hybrid MCDM method for sustainability. Sustainability (Switzerland), v. 6, p. 217-235, 2014.

DOI: $10.3390 /$ su6010217

ZHOU, R.; MA, X.; LI, S.; LI, J. The Green Supplier Selection Method For Chemical Industry With Analytic Network Process And Radial Basis Function Neural Network. International Journal on Advances in Information Sciences and Service Sciences, v. 4, n. 4, p. 147158, 2012. DOI: $\underline{10.4156 / \text { AISS.vol4.issue } 4.18}$

ZSIDISIN, G. A.; SIFERD, S. P. Environmental purchasing: a framework for theory development. European Journal of Purchasing and Supply Management, v. 7, p. 61-73, 2001. DOI: $\underline{10.1016 / S 0969-7012(00) 00007-1}$

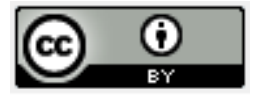

Artigo recebido em 15/02/2016 e aceito para publicação em 10/03/2016

DOI: http://dx.doi.org/ 10.14488/1676-1901.v16i4.2357 


\section{APÊNDICE}

Quadro 3 - Lista de trabalhos resultantes do processo de revisão sistemática

\begin{tabular}{|c|c|c|c|c|c|}
\hline № & Nome do Artigo & Autores & Ano & Palavras-chave & Journal \\
\hline 1 & $\begin{array}{l}\text { A green supplier selection } \\
\text { model for high-tech industry }\end{array}$ & Lee et al. & 2009 & $\begin{array}{c}\text { Analytic hierarchy process; } \\
\text { Environment; Fuzzy set theory; } \\
\text { FEAHP; Green supplier } \\
\text { Fuzzy set theory. FEAHP. Green } \\
\text { supplier }\end{array}$ & $\begin{array}{l}\text { Expert Systems with } \\
\text { Applications }\end{array}$ \\
\hline 2 & $\begin{array}{l}\text { Integrating sustainability into } \\
\text { supplier selection with grey } \\
\text { system and rough set } \\
\text { methodologies }\end{array}$ & Bai e Sarkis & 2010 & $\begin{array}{l}\text { Environment/ Sustainability Supply } \\
\text { chain/ Rough set/ Grey system }\end{array}$ & $\begin{array}{l}\text { International Journal } \\
\text { of Production } \\
\text { Economics }\end{array}$ \\
\hline 3 & $\begin{array}{l}\text { Integration of artificial neural } \\
\text { network and MADA methods } \\
\text { for green } \\
\text { supplier selection }\end{array}$ & $\begin{array}{l}\text { Kuo, Wang e } \\
\text { Tien }\end{array}$ & 2010 & $\begin{array}{l}\text { Green supplierselection } \\
\text { Data envelopmentanalysis } \\
\text { Analytic networkprocess } \\
\text { Artificial neuralnetwork }\end{array}$ & Cleaner Production \\
\hline 4 & $\begin{array}{l}\text { A novel fuzzy multi-criteria } \\
\text { decision framework for } \\
\text { sustainable supplier selection } \\
\text { with incomplete information }\end{array}$ & $\begin{array}{l}\text { Büyüközkan e } \\
\text { Çifçi }\end{array}$ & 2011 & $\begin{array}{c}\text { Sustainable supply chain/ Supplier } \\
\text { selection/ Analytic network process/ } \\
\text { Fuzzy logic/ Incomplete preference } \\
\text { relations }\end{array}$ & $\begin{array}{l}\text { Computers in } \\
\text { Industry }\end{array}$ \\
\hline 5 & $\begin{array}{l}\text { The green supplier selection } \\
\text { method for chemical industry } \\
\text { with analytic network process } \\
\text { and radial basis function } \\
\text { neural network }\end{array}$ & Zhou et al. & 2012 & $\begin{array}{c}\text { Green Supplier Selection; Analytic } \\
\text { Network Process (ANP); Radial Basis } \\
\text { Function } \\
\text { (RBF); Neural Network; Chemical } \\
\text { Industry }\end{array}$ & $\begin{array}{l}\text { Advances in } \\
\text { Information Sciences } \\
\text { and Service } \\
\text { Sciences }\end{array}$ \\
\hline 6 & $\begin{array}{l}\text { Sustainable supplier selection: } \\
\text { A ranking model based on } \\
\text { fuzzy inference system }\end{array}$ & $\begin{array}{l}\text { Amindoust et } \\
\text { al. }\end{array}$ & 2012 & $\begin{array}{c}\text { Sustainable supplier selection/ Supply } \\
\text { chain management/ Fuzzy inference } \\
\text { system }\end{array}$ & $\begin{array}{l}\text { Applied Soft } \\
\text { Computing Journal }\end{array}$ \\
\hline 7 & $\begin{array}{l}\text { Integrated fuzzy multi criteria } \\
\text { decision making method and } \\
\text { multiobjective } \\
\text { programming approach for } \\
\text { supplier selection and order } \\
\text { allocation in a green supply } \\
\text { chain }\end{array}$ & Kannan et al. & 2013 & $\begin{array}{l}\text { Green supply chain management } \\
\text { (GSCM) } \\
\text { Supplier selection } \\
\text { Multi-objective linear programming } \\
\text { (MOLP) } \\
\text { Maxi-min method } \\
\text { Order allocation }\end{array}$ & Cleaner Production \\
\hline 8 & $\begin{array}{c}\text { Using DEMATEL to develop a } \\
\text { carbon management model of } \\
\text { supplier } \\
\text { selection in green supply chain } \\
\text { management }\end{array}$ & Hsu et al. & 2013 & $\begin{array}{c}\text { Carbon management, } \\
\text { Supplier selection, } \\
\text { Green supply chain management, } \\
\text { DEMATEL }\end{array}$ & Cleaner Production \\
\hline 9 & $\begin{array}{l}\text { Selection ideal coal suppliers } \\
\text { of thermal power plants using } \\
\text { the matter-element extension } \\
\text { model with integrated } \\
\text { empowerment method for } \\
\text { sustainability }\end{array}$ & Tan et al. & 2014 & não disponível & $\begin{array}{l}\text { Mathematical } \\
\text { Problems in } \\
\text { Engineering }\end{array}$ \\
\hline 10 & $\begin{array}{l}\text { A decision support tool for } \\
\text { sustainable supplier selection } \\
\text { in manufacturing firms }\end{array}$ & Orji e Wei & 2014 & $\begin{array}{c}\text { supplier selection, sustainability, } \\
\text { Original Equipment Manufacturers } \\
\text { (OEM }\end{array}$ & $\begin{array}{c}\text { Industrial } \\
\text { Engineering and } \\
\text { Management }\end{array}$ \\
\hline 11 & $\begin{array}{l}\text { A new fuzzy TOPSIS-TODIM } \\
\text { hybrid method for green } \\
\text { supplier selection using fuzzy } \\
\text { time function }\end{array}$ & $\begin{array}{l}\text { Arshadi } \\
\text { Khamseh e } \\
\text { Mahmoodi }\end{array}$ & 2014 & não disponível & $\begin{array}{l}\text { Advances in Fuzzy } \\
\text { Systems }\end{array}$ \\
\hline
\end{tabular}




\begin{tabular}{|c|c|c|c|c|c|}
\hline № & Nome do Artigo & Autores & Ano & Palavras-chave & Journal \\
\hline 12 & $\begin{array}{l}\text { Selecting green suppliers } \\
\text { based on GSCM practices: } \\
\text { Using fuzzy TOPSIS } \\
\text { applied to a Brazilian } \\
\text { electronics company }\end{array}$ & $\begin{array}{l}\text { Kannan, De } \\
\text { Sousa Jabbour } \\
\text { e Jabbour }\end{array}$ & 2014 & $\begin{array}{c}\text { Green supply chain management } \\
\text { (GSCM) } \\
\text { Green supplier selection } \\
\text { Fuzzy set theory } \\
\text { TOPSIS } \\
\text { Triangular fuzzy number }\end{array}$ & $\begin{array}{l}\text { EUROPEAN } \\
\text { JOURNAL OF } \\
\text { OPERATIONAL }\end{array}$ \\
\hline 13 & $\begin{array}{c}\text { Selecting green supplier of } \\
\text { thermal power equipment by } \\
\text { using a hybrid MCDM method } \\
\text { for sustainability }\end{array}$ & Zhao e Guo & 2014 & $\begin{array}{l}\text { Fuzzy-entropy; Fuzzy-TOPSIS; } \\
\text { Green supplier selection; } \\
\text { Sustainability; Thermal power } \\
\text { equipment }\end{array}$ & $\begin{array}{l}\text { Sustainability } \\
\text { (Switzerland) }\end{array}$ \\
\hline 14 & $\begin{array}{l}\text { Green supplier selection and } \\
\text { evaluation using DEA-type } \\
\text { composite indicators }\end{array}$ & $\begin{array}{l}\text { Dobos e } \\
\text { Vörösmarty }\end{array}$ & 2014 & $\begin{array}{c}\text { Green supplierassessment } \\
\text { DEA } \\
\text { Common weightsanalysis } \\
\text { Composite indicators } \\
\text { Multicriteria decisionmaking }\end{array}$ & $\begin{array}{l}\text { International Journal } \\
\text { of Production } \\
\text { Economics }\end{array}$ \\
\hline 15 & $\begin{array}{l}\text { Evaluating a green supplier } \\
\text { selection problem using a } \\
\text { hybrid MODM algorithm }\end{array}$ & Bakeshlou et al. & 2014 & $\begin{array}{c}\text { Green supplier selection · Fuzzy } \\
\text { analytical network process (ANP) } \\
\text { Fuzzy decision making trial and } \\
\text { Evaluation laboratory (DEMATEL) · } \\
\text { Order allocation . Fuzzy multi- } \\
\text { objective linear programming (MOLP) } \\
\text { Weighted max-min operator } \\
\text { Wer }\end{array}$ & $\begin{array}{c}\text { Intelligent } \\
\text { Manufacturing }\end{array}$ \\
\hline 16 & $\begin{array}{l}\text { An integrated MCDM approach } \\
\text { to green supplier selection }\end{array}$ & Yazdani & 2014 & $\begin{array}{l}\text { AHP; Environmental factors; Fuzzy } \\
\text { TOPSIS; MCDM; Supplier selection }\end{array}$ & $\begin{array}{l}\text { International Journal } \\
\text { of Industrial } \\
\text { Engineering } \\
\text { Computations }\end{array}$ \\
\hline 17 & $\begin{array}{c}\text { A hybrid multiple criteria group } \\
\text { decision-making approach for } \\
\text { green supplier selection in the } \\
\text { TFT-LCD industry }\end{array}$ & Tsui e Wen & 2014 & não disponível & $\begin{array}{l}\text { Mathematical } \\
\text { Problems in } \\
\text { Engineering }\end{array}$ \\
\hline 18 & $\begin{array}{l}\text { A decision support system to } \\
\text { select suppliers for a } \\
\text { sustainable supply chain } \\
\text { based on a systematic DEA } \\
\text { approach }\end{array}$ & Shi et al. & 2014 & $\begin{array}{c}\text { Decision support system; Green } \\
\text { procurement; Green supplier } \\
\text { evaluation; Green supplier selection; } \\
\text { Sustainable supply chain; Systematic } \\
\text { DEA approach }\end{array}$ & $\begin{array}{l}\text { Information } \\
\text { Technology and } \\
\text { Management }\end{array}$ \\
\hline 19 & $\begin{array}{c}\text { An intuitionsitic fuzzy judement } \\
\text { matrix and TOPSIS integrated } \\
\text { multi-criteria decision making } \\
\text { for green supplier selection }\end{array}$ & $\begin{array}{l}\text { Cao, Wu e } \\
\quad \text { Liang }\end{array}$ & 2015 & $\begin{array}{l}\text { Green supplier selection, multicriteria } \\
\text { decison making, intuitionsitic fuzzy } \\
\text { number, TOPSIS method, subjective } \\
\text { weights, objective weights }\end{array}$ & $\begin{array}{l}\text { Journal of Intelligent } \\
\text { \& Fuzzy Systems }\end{array}$ \\
\hline 20 & $\begin{array}{l}\text { Green suppplier selection } \\
\text { using na AHP-Entropy- } \\
\text { TOPSIS framework } \\
\end{array}$ & $\begin{array}{l}\text { Freeman e } \\
\text { Chen }\end{array}$ & 2015 & green issues, AHP, modeling & $\begin{array}{c}\text { Supply Chain } \\
\text { Management: An } \\
\text { International Journal }\end{array}$ \\
\hline 21 & $\begin{array}{c}\begin{array}{c}\text { Supplier selection problems in } \\
\text { fashion business operations } \\
\text { with sustainability } \\
\text { considerations }\end{array} \\
\end{array}$ & Jia et al. & 2015 & $\begin{array}{c}\text { Fashion industry; Multi-criteria } \\
\text { decision making; Supplier selection; } \\
\text { Sustainability; TOPSIS }\end{array}$ & $\begin{array}{l}\text { Sustainability } \\
\text { (Switzerland) }\end{array}$ \\
\hline 22 & $\begin{array}{c}\text { Supplier selection for } \\
\text { sustainable operations: a triple } \\
\text { bottom line approach using } \\
\text { bayesian framework }\end{array}$ & $\begin{array}{l}\text { Sarkis e } \\
\text { Dhavele }\end{array}$ & 2015 & $\begin{array}{l}\text { supplier selection, multiple objectives, } \\
\text { triple bottom line, bayesian } \\
\text { framework, markov chain monte } \\
\text { carlos simulation, Gibbs sampler }\end{array}$ & $\begin{array}{l}\text { International Journal } \\
\text { of Production } \\
\text { Economics }\end{array}$ \\
\hline 23 & $\begin{array}{l}\text { An innovative integration of } \\
\text { fuzzy logic and systems } \\
\text { dynamics in sustainable } \\
\text { supplier selection: a case on } \\
\text { manufactuting industry }\end{array}$ & Orji e Wei & 2015 & $\begin{array}{l}\text { Fuzzy logic, supplier selection, } \\
\text { sustainability, system dynamics }\end{array}$ & $\begin{array}{l}\text { Computers \& } \\
\text { Industrial } \\
\text { Engineering }\end{array}$ \\
\hline
\end{tabular}




\begin{tabular}{|c|c|c|c|c|c|}
\hline № & Nome do Artigo & Autores & Ano & Palavras-chave & Journal \\
\hline 24 & $\begin{array}{c}\text { Fuzzy Axiomatic Design } \\
\text { approach based green supplier } \\
\text { selection: acase study from } \\
\text { Singapore }\end{array}$ & $\begin{array}{l}\text { Kannan, } \\
\text { Govindan e } \\
\text { Rajendran, }\end{array}$ & 2015 & $\begin{array}{l}\text { Green supplier selection } \\
\text { Multi criteria decision making } \\
\text { Fuzzy Axiomatic Design } \\
\text { Environmental performance }\end{array}$ & Cleaner Production \\
\hline 25 & $\begin{array}{l}\text { An integrated green supplier } \\
\text { selection approach with } \\
\text { analytic network process and } \\
\text { improved Grey relational } \\
\text { analysis }\end{array}$ & $\begin{array}{l}\text { Hashemi, } \\
\text { Karimi e } \\
\text { Tavana }\end{array}$ & 2015 & $\begin{array}{c}\text { Green supply chain } \\
\text { management(GSCM), } \\
\text { Environmental sustainability, } \\
\text { Supplier selection, } \\
\text { Analytic network process(ANP), } \\
\text { Improved Grey relation alanalysis } \\
\text { (GRA), } \\
\text { Grey theory }\end{array}$ & $\begin{array}{l}\text { Production } \\
\text { Economics }\end{array}$ \\
\hline 26 & $\begin{array}{l}\text { Developing a green supplier } \\
\text { selection model by using the } \\
\text { DANP with VIKOR }\end{array}$ & Kuo, Hsue Li & 2015 & $\begin{array}{l}\text { DANP; EICC; Environmental } \\
\text { performance; Supplier selection; } \\
\text { VIKOR }\end{array}$ & $\begin{array}{l}\text { Sustainability } \\
\text { (Switzerland) }\end{array}$ \\
\hline 27 & $\begin{array}{c}\text { Identifying Robust portfolios of } \\
\text { suppliers: a sustainability } \\
\text { selectionand development } \\
\text { perspective }\end{array}$ & Trapp e Sarkis & 2015 & $\begin{array}{l}\text { Sustainability/ Green supply chain } \\
\text { management/ Supplier selection/ } \\
\text { Supplier development/ Integer } \\
\text { programming/ Optimization }\end{array}$ & Cleaner Production \\
\hline
\end{tabular}

